relating to the renal system in gynæcology, and the third dealing with symptoms relating to the gastro-intestinal tract in gynæcology. Displacements are then considered, followed by infection, and after a chapter on extra-uterine pregnancy, almost 120 pages are devoted to the important subject of new growths. Then comes a discussion on developmental errors, and finally a section on operative gynæcology, which is quite properly, in a volume of this kind, considered briefly. The outline above will show that the scope of the work is comprehensive while it is essentially clinical and practical. As regards the various aspects of the book and of the views expressed there, criticism is very difficult as a strong vein of common sense, with the acceptance of generally recognised views, runs throughout the work, so that it can meet with only general approval. Each chapter has been carefully revised and brought up to date in view of the most recent advances, and in this respect the chapter dealing with the ever changing subject of endocrinology is well balanced and complete. A good index of a work of this kind lies in the views expressed on such a subject as retroversion of the uterus, and there the reader will find a very fair balance of opinion and especially the valuable advice that in the presence of retroversion, symptoms are so often due, not to that condition, but to some associated condition, such as salpingitis.

One slight criticism may be made in the description of the symptoms of tubal gestation, in which, as so often happens, the dramatic symptoms of intra-peritoneal hæmorrhage are emphasized rather at the expense of the large proportion of cases in which the symptoms are much less acute and in which the condition may be mistaken for salpingitis or almost any other gynæcological lesion. But this is only a detail, and in general the book can be recommended to the undergraduate and to the postgraduate as a faithful mirror of British gynæcological teaching and practice of to-day, and if the practitioner is guided by the principles laid down there he will carry on his practice of this subject at a high level and in the best interests of his patients.

The format of the book is good and the glazed paper and good type make comfortable reading, while the illustrations, most of which are in line, are expressive and well chosen.

\section{A TEXT-BOOK OF MIDWIFERY FOR STUDENTS AND PRACTITIONERS.}

By Prof. R. W. Johnstone, C.B.E., M.A., M.D., F.R.C.S.E., etc. 10th Edition. A. \& C. Black, Ltd. Price 18s. Od.

This popular text-book of midwifery now reaches its tenth edition. Every chapter has been subjected to a critical revision and certain sections have been somewhat expanded in the light of recent research, especially those on the hormonic function of the ovary, disease in association with midwifery, puerperal infection, infant feeding, the heart, diabetes and glycosuria. The new illustrations include four remarkable cineradiographs illustrating the fœtal circulation and no less than four additional plates in full colour to illustrate placental circulation, the liver in eclampsia, placental infarcts and concealed accidental hæmorrhage.

The general lay-out of the work conforms to that of the previous editions and the chapter on puerperal infection is an excellent exposition. The section devoted to the newborn child is outstanding and in the last fifty pages the author outlines the essentials of all the obstetric operations in a clear concise form.

This work will continue to retain its popularity. It is well produced, clearly written and well illustrated.

\section{THE SEXUAL PERVERSIONS AND ABNORMALITIES.}

By Clifford Allen, M.D., M.R.C.P., D.P.M.

Pp. 200. Oxford Medical Publications. 1940. Price $7 \mathrm{~s} .6 \mathrm{~d}$.

It is strange that with all that is written about sex perversions, and in view of the pre-occupation of the Freudian school with sex problems, that the need for what might be termed a text book on the subject giving a general survey of the whole problem has been left so long unsatisfied. This excellent book satisfies that need. The earlier pre-Freudian books were little else than clinical descriptions of the perversions with the minimum of psychopathology. The Freudian school remedied this and dealt with the deep causal factors, but its descriptions were too bound up with a particular theory to give the general reader a sound knowledge of the subject.

For the practitioner who desires an up-to-date account of the clinical types of perversion together with a concise account of their psychopathology. no better book can be recommended than this one.

After dealing with the instincts in relation to sexual abnormalities, and with sexual abnormalities in animals, the author deals with the normal stages of sexual development in man. This leads on to a classification of the sexual abnormalities. according to the phases of an instinctive action. namely the stimulus (e.g. fetichism), the mode of expression (e.g. sadism), the strength of the urge (e.g. impotence nymphomania), and the object (homo-sexuality). The book concludes with a description of representative cases.

This book is clearly written, and commendably brief. It deals concisely with subjects with which the usual practitioner should in these days be conversant, which he will often meet in his practice if he has the real confidence of his patients, but which unfortunately are rarely dealt with in the medical schools. Dr. Allen's experience and research work on the physiological aspects of sexual abnormalities gives him a balance in dealing with these subjects not always possessed by those whose knowledge and experience is confined to the psychological aspect alone. 